\title{
Contar e narrar os desastres ${ }^{1}$
}

\section{Sandrine Revet ${ }^{2}$}

Resumo: Desde os anos 80, a progressiva institucionalização de um governo mundial dos desastres se baseia em um conjunto de números compilados em bases de dados. $\mathrm{O}$ artigo se interessa por duas dentre elas: EM-DAT, produzida pelo CRED; e Desinventar, produzida pela La Red. Cada uma destas bases de dados elabora, ao seu modo, e com a ajuda de traduções, uma narrativa sobre os desastres, e um tipo de diálogo se estabelece entre elas, que nos falam das diferentes dimensões de um mundo "desastroso".

Palavras-Chave: desastres; narrativas; EM-DAT; desinventar

Abstract: Since the 1980s, the gradual institutionalization of a World government for disasters was based on a set of figures compiled in databases. The article focuses on two of them: EM-DAT, produced by CRED, and Desinventar, produced by La Red. Each one produces, in its own way and by means of translations, a narrative of disasters and a kind of dialogue is established between them, which illustrates well the different dimensions of the "disastered" world.

Keywords: disasters; narrative; EM-DAT; desinventar

1 Tradução do artigo "Compter et raconter les catastrophes" publicado originalmente na revista Communications, n. 96, p. 81-92, 2015, École des Hautes Études en Sciences Sociales - Centre Edgar Morin. Disponível em: <https://bit.ly/2MzYjcY>.

2 Sandrine Revet é antropóloga, Diretora de Pesquisas no CERI (Centro de Estudos e de Investigações Internacionais) - SciencesPo, em Paris. Especializada em antropologia dos desastres, ela é cofundadora da "Association pour la recherche sur les catastrophes et les risques en anthropologie (ARCRA) e da Rede DICAN (Disaster and Crisis anthropological Network) na "European Association for Social Anthropologists" (EASA).Email: <sandrine.revet@sciencespo.fr>. 
Nenhum fenômeno natural - furacão, tsunami, terremoto ou erupção vulcânica - se torna um "desastre", sem uma narrativa capaz de sustentá-lo. Esta narrativa, frequentemente composta por uma multidão de vozes, utiliza uma base dramática, que combina diferentes ingredientes: i) narrativas das pessoas afetadas pelo que lhes aconteceu "naquele dia";ii) imagens de destruição, que colocam em cena a natureza e as construções humanas desordenadas, despedaçadas, sujas, reviradas; e, finalmente, iii) os números, que tentam apreender - e dar - a medida do acontecimento. É por estes números que me interesso aqui. Pois contar os desastres é narrar sobre alguma coisa.

Seja quem for seu produtor - habitantes, autoridades locais ou nacionais, especialistas, mídia, instâncias internacionais... -, o cálculo dos desgastes e o número de vítimas têm um lugar central nas narrativas dos desastres. Os números podem servir para diversas causas: adaptar os meios, até então existentes, para enfrentar o acontecimento e ajudar a sociedade atingida; emocionar o leitor distante para tentar mobilizá-lo; assegurar às próprias vítimas de que elas viveram um drama digno de interesse; conduzir, enfim, o acontecimento à sua dimensão social, ao torná-lo comparável a outros, tanto para convencer de que ele é excepcional, ou, ao contrário, de que ele é bastante rotineiro. Pensar um desastre, a partir da mensuração de suas consequências, é trazê-lo à sua dimensão social: número de casas destruídas; quilômetros de estradas atingidos; quantidade de hospitais tornados impraticáveis; e, certamente, o número de feridos ou de vítimas. Toda esta contabilidade fala de um mundo social afetado, transtornado, perturbado pelo acontecimento, e que se torna um "desastre" desde o momento em que os números permitem confirmá-lo como tal.

Desde os anos 70, os desastres ditos "naturais [1]", que são, até aquele momento, objeto da atenção internacional, centrada unicamente no socorro ou na assistência humanitária quando o acontecimento já ocorreu, são progressivamente também objeto da atenção de atores e de instituições internacionais, preocupados em reduzir os seus impactos, quer dizer, interessados na prevenção [2]. Para a mobilização desta questão, no primeiro momento, foi necessária a produção de uma narrativa universalmente audível sobre os desastres e suas consequências. Esta narrativa é notadamente apoiada na contagem, em escala mundial, 
dos desastres e de seus impactos, e sobre a implementação de ferramentas para a acumulação de números, até então, ou inexistentes, ou dispersos, ou limitados às escalas locais ou nacionais.

A constituição das bases de dados, a partir do levantamento do conjunto de desastres ocorridos na escala planetária, é um dos instrumentos do governo mundial das catástrofes, o qual emerge a partir dos anos 1980 para tentar estandardizar e coordenar os recursos mobilizados para estes acontecimentos, que se tornam custosos e mais "escandalosos", aos olhos da opinião mundial, mais rapidamente alertada pela mídia. O processo de quantificação internacional, que apareceu nos anos 1970 e se consolidou nos anos 1980, logo conduziu à criação de duas grandes bases: a EM-DAT, coordenada por um centro de pesquisa da Universidade Católica de Louvain-la-Neuve; e a NatCatSERVICE, constituída a partir dos arquivos da seguradora Munich Re, em 1974. Durante muito tempo, estas bases foram as únicas produtoras de dados sobre desastres em escala internacional. Só recentemente, outros projetos de quantificação surgiram, encorajados notadamente pelas Nações Unidas, em torno do Quadro de Ação de Hyogo, que engaja os países signatários a por em prática medidas de redução da vulnerabilidade e dos riscos. Hoje, existem cinco bases internacionais de dados sobre os desastres e um número crescente de bases nacionais [3].

Neste artigo, interesso-me por duas, dentre elas; a EM-DAT [4], produzida e alimentada pelo Centro de Pesquisa sobre epidemiologia dos desastres (CRED), da Universidade Católica de Louvain-la-Neuve; e a Desinventar [5], uma base de dados regionais, criada pela Rede Latino-Americana de pesquisas em ciências sociais sobre desastres (La Red), alimentada por uma ONG colombiana (Corporación OSSO). Cada uma, a seu modo, constitui uma referência na paisagem de quantificação e de big datas sobre os desastres. Todas as duas bases são mantidas e promovidas pela agência das Nações Unidas, responsável pela temática da “redução de riscos e desastres”, a UNISDR (United Nations International Strategy for Disaster Risk Reduction). Ambas são de acesso público e gratuito e contribuem para a produção de dados, utilizados no relatório bienal das Nações Unidas sobre os desastres, o Global Assessment Report [6], referência no tema. Elas ocupam um papel central no governo mundial dos desastres. Além disso, 
participam da emergência de narrativas necessárias ao reconhecimento dos desastres como problemas públicos em escala internacional [7].

A particularidade destas narrativas é a de se apoiarem na acumulação de dados. Não são mais os números de um desastre em particular que são pertinentes, e sim o somatório de todos os desastres, que ocorreram em certo período, ou em certa região do mundo; ou, ainda, os desgastes produzidos por certo tipo de fenômeno, em comparação com um outro (terremotos versus inundações, por exemplo). A narrativa assim produzida não é mais a de um desastre, e sim a de um mundo "desastroso". Entretanto, mesmo se uma e outra falam de "desastres”, estas duas bases de dados não estão situadas na mesma escala, não contam as mesmas coisas e, ao final, produzem narrativas muito diferentes.

\section{EM-DAT: Big Data e grandes desastres}

A base de dados EM-DAT, que é hoje uma referência internacional, foi criada em 1983 pelo Centro de Pesquisa sobre epidemiologia de desastres de Louvain-la-Neuve, na Bélgica. Esta ideia de epidemiologia dos desastres emerge a partir dos anos 1971-1972 na Bélgica, no espírito do diretor da Escola de Saúde Pública da Universidade Católica de Louvain, o epidemiologista Michel Lecha, que é formado na Universidade Johns Hopkins e trabalhou durante muitos anos na África com a questão da lepra. Para ele,trata-se de desenvolver, diante dos desastres, ainda quase exclusivamente centrados nas abordagens das ciências da terra e dos estudos das ameaças naturais, uma outra abordagem, que colocasse em primazia os "fatores sociais", de forma a contribuir para tornar os acontecimentos naturais ainda mais devastadores. A posteriori, Michel Lechat justifica assim:

Se a epidemiologia é o estudo dos fenômenos da saúde nas populações, não existe nenhuma razão para que os desastres, surpreendendo comunidades inteiras e fazendo às vezes milhares de vítimas, ou mesmo centenas de milhares, não sejam investigados pelos epidemiologistas em uma perspectiva de prevenção, de assistência e de redução de danos em termos de mortes, de doenças e de invalidez [8]. 
No primeiro momento, por conseguinte, a epidemiologia considera o desastre tal como a doença, notadamente na área da saúde, pelo o número de mortes que ele provoca, em função de diferentes determinantes, para tentar antever os fatores que permitiriam reduzir estes impactos.

Mas depois da saída de Michel Lechat, a nova diretora do CRED também epidemiologista -, Debby Guha-Sapir, lança o projeto que faria deste Centro a fonte nodal de informação sobre os desastres no mundo. O projeto de quantificação internacional dos desastres se instala com uma base de dados internacional, EM-DAT (Emergency Disasters Database) [9]. Esta base, inicialmente em grande parte financiada pelo Departamento de Assistência Internacional do governo americano (OFDA/USAID [10]), que era então a agência implicada financeiramente nas operações de socorro ao nível internacional e que buscava racionalizar sua ação, hoje compila dados sobre mais de dezenove mil desastres ocorridos no mundo desde 1900. O CRED utiliza fontes produzidas prioritariamente pelas Nações Unidas, depois pela OFDA, pelos governos, e, enfim, pela Federação Internacional das Sociedades da Cruz Vermelha e do Crescente Vermelho [11]. Os dados provenientes das organizações não governamentais, das companhias de seguro, dos institutos de pesquisa e das agências de notícias são também mobilizados, entretanto, com maior atenção sobre a sua qualidade. Esta hierarquização é justificada pelo CRED pela "qualidade das fontes" e também pelas "questões políticas", que podem afetar os números - pressupondo, por isso, que aqueles provenientes das Nações Unidas não seriam, ou seriam menos, sujeitos a erros [12].

Os três objetivos anunciados desta base de dados são a assistência à ação humanitária, a "racionalização" da tomada de decisão na preparação para desastres e o oferecimento de uma "base objetiva" para a avaliação da vulnerabilidade [13]. Para o EM-DAT, os desastres pertinentes são aqueles que produziram "ao menos 10 mortes ou 100 pessoas afetadas" e que foram objetos de uma demanda de assistência internacional ou de uma declaração de estado de urgência, em nível internacional. Assim que um acontecimento entra em uma base de dados, ele obtém um "número de desastre" e catorze campos devem ser informados, para que ele seja documentado devidamente. Seis campos são constituídos por 
informações sobre o país, a data de ocorrência, assim como as características do desastre (tipo de fenômeno). Além deles, sete campos devem informar os danos produzidos: número de mortos, de feridos, de desalojados, de pessoas atingidas e de custo estimado dos prejuízos. Um campo suplementar permite fornecer outros tipos de informação de tipo geográfico, medida do desastre (por exemplo, a magnitude dos terremotos ou a força dos furacões...), seu estatuto diante da ajuda internacional ou o montante dos auxílios.

As traduções destes dados adquirem a forma de mapas, de quadros e de gráficos. Porém, o uso interativo da base permite a cada usuário criar assuas próprias traduções, caso ele não deseje usar os mapas e quadros já existentes e disponíveis on-line. A tradução, sem qualquer dúvida, a mais procurada, é o relatório anual assinado pela diretora do CRED e sua equipe. Este relatório apresenta os números anuais, em cerca de cinquenta páginas, com as classificações sob a forma dos "Top 10": os desastres mais mortíferos, os países mais atingidos, as regiões mais afetadas... e alguns gráficos que passam a circular logo a seguir, em numerosos fóruns políticos e científicos.

Esta ferramenta de quantificação constitui um serviço indispensável para as agências internacionais,por ser ela utilizada tanto para orientar, quanto para legitimar as suas ações. Nessa perspectiva, a Organização Mundial da Saúde, a Federação Internacional da Cruz Vermelha, a USAID e a Estratégia Internacional da ONU de Prevenção de Desastres (UNISDR) contribuem, em parte, também para o seu financiamento, prova de que tais agências precisam dessa ferramenta. A escala escolhida para formar a base é nacional, e não local, o que torna a compreensão dos fatores sociais de vulnerabilidade pouco precisa, mas que corresponde à escala de tomada de decisões e, sobretudo, de legitimação dos atores internacionais e humanitários. Pode-se, a partir dela, sensibilizar um governo sobre os riscos que ameaçam oseu país, ou proceder a abertura de um programa sobre desastres, sem, no entanto, adquirir um conhecimento mais fino sobre os mecanismos e os fatores que contribuem para agravar as consequências dos desastres, quando eles acontecem.

Com o EM-DAT, o CRED se tornou o principal produtor de estatísticas internacionais sobre desastres, e seus dados são citados na introdução de 
todos os relatórios das agências da $\mathrm{ONU}$, das grandes organizações não governamentais e em numerosos artigos científicos de pesquisa sobre desastres "naturais". Assim, esta base de dados se afasta dos objetivos anunciados no início da epidemiologia dos desastres de Lechat, que buscava uma melhor compreensão dos desastres e de seus fatores agravantes, para ir em direção ao desenvolvimento de um serviço para as grandes agências internacionais.

\section{Desinventar: tornar visível os "pequenos desastres"}

Em 1993, La Red, uma rede de pesquisa latino-americana, coloca em atuação a sua própria base de dados, Desinventar, apresentada como "alternativa" àquelas utilizadas pelas grandes instâncias internacionais e notadamente à base do CRED. Para estes pesquisadores - geógrafos, urbanistas, antropólogos, historiadores - que se autodesignam como "radicais [14]", a ideia é provar que contar não é uma operação neutra, uma vez que ela implica em escolhas de ordem política, que influenciam no modo de compreensão dos desastres. Os pesquisadores da La Red têm constantemente "desnaturalizado" os desastres [15], destacando os fatores sociais, políticos e econômicos que os tornam destrutivos. Nesta perspectiva, a prioridade deve ser dada não sobre a compreensão da ameaça natural, mas sobre a vulnerabilidade da sociedade atingida por ela. É o encontro do fenômeno natural e das condições de vulnerabilidade que produz o desastre.

O projeto Desinventar reivindica uma mudança de escala e um deslizamento de objeto, em relação à base EM-DAT. Com ele, La Red pretende defender um outro modo de fazer a sistematização e a quantificação dos dados dos desastres. Segundo os seus iniciadores, a abordagem deve ser resolutamente local. Além disso, é preciso se interessar por todos os tipos de desastres e não considerar somente o limite mínimo do número de mortes ou da quantidade de danos sofridos. Devem ser contabilizados, também, tempestades de granizo, poluição olfativa, devido à incineração de resíduos em céu aberto e pelos ventos fortes, bem como os desmoronamentos de muitas casas em um bairro, depois de fortes chuvas. Desinventar reúne hoje mais de vinte e três mil entradas e contém informações dos desastres ocorridos depois de 1914, em grande parte na América 
Latina. Além disso, o campo se alarga cada vez mais, pois a base exibiu, em 2012, as contribuições de dezenove países latino-americanos, treze países do Oriente Médio e da Ásia,além de cinco países africanos [16].

Os dados históricos são provenientes majoritariamente dos arquivos de jornais, nacionais e locais. As informações mais recentes condensam fontes vindas da mídia e são igualmente alimentadas por bases de dados nacionais dos sistemas de defesa civil, desde 2007, sendo elas alimentadas por informações fornecidas por: polícias nacionais e locais, bombeiros, sociedades nacionais da Cruz Vermelha e outros atores que intervêm em casos de desastres. Desinventar permite atuar na escala da cidade, do bairro ou do distrito. Esta escolha torna possível, contrariamente à escala nacional privilegiada pelo EM-DAT, trabalhar os fatores agravantes da vulnerabilidade. Com esta base, também é possível, aos usuários, compilar os seus próprios dados e realizar as suas próprias traduções - mapas, curvas, quadros e gráficos - graças a um programa gratuito disponível online.

Desinventar conseguiu se tornar uma base de referência nas instâncias internacionais e hoje em dia é promovida e, em parte também, financiada pelo ISDR. Pode-se explicar esta ascensão particular de uma "pequena" base de dados regional pelos laços que unem os seus iniciadores a certos quadros do ISDR, igualmente originados de La Red e que alcançaram postos na governança internacional. Sensíveis à questão da prevenção, e por consequência à temática da vulnerabilidade, muitos personagens centrais desta agência da ONU tentam promover a perspectiva das ciências sociais "radicais" que La Red reivindica.

\section{Estandardizar para estabilizar as disputas?}

Uma primeira reflexão sobre estas bases de dados consiste, como já fizeram Cloé Vallette e Stéphane Cartier, em uma perspectiva comparada, nos seus respectivos trabalhos de campona Algéria e na Costa Rica, em mostrar que os números disponíveis nas bases de dados são o resultado de lutas e de discussões e não uma simples "coleta" de dados [17]- a propósito, mais corretamente dito "obtidos", por Bruno Latour [18]. Trata-se, então, de mostrar as condições de 
produção destes números, que, nos dois casos,procedem de escolhas e de disputas [19]. Frequentemente polêmicos - notadamente em torno do número de vítimas -, os dados nascem após os desastres, e os números que chegam a entrar nas bases internacionais são aqueles que estabilizam provisoriamente uma disputa, permitindo aos muitos atores envolvidos produzir uma narrativa satisfatória sobre o desastre [20], como igualmente dar a ela uma representação "racional" porque codificada - dos acontecimentos de outro modo incomensuráveis [21].

A produção de dados estandardizados não se coloca sem problemas. Desinventar, que se apoia em fontes locais - e frequentemente vindas da imprensa -, encontra dificuldades para nomear, depois classificar os acontecimentos e estandardizar as maneiras de proceder em diferentes países interessados no uso da ferramenta. É preciso, de fato, ocorrer um acordo sobre os casos sempre particulares e suscetíveis de serem discutidos: por exemplo, como categorizar a inundação de um bairro, devido à ruptura de uma canalização? De fato, se sua origem é claramente antrópica, o termo "inundação" reenvia mais a um fenômeno natural [22]. A questão pouco importa, quanto às consequências, no que se refere aos impactos de um só fenômeno, mas, ao contrário, a entrada em uma base de dados, que resulta não somente na possibilidade de adição, mas também na de comparação e de análise, e mesmo na de projeção, é acompanhada de seu pacote de incertezas, de vieses e de questões que as duas bases de dados estão longe de resolver. Mas decorre destes dados, como de todas as estatísticas - que se utilizam da força dos grandes números para apagar as imprecisões que contêm -, a linguagem probabilística, que permite precisar o grau de confiança que se pode dar a estas medidas [23].

\section{Modos dramatúrgicos e narrativas do mundo desastre}

Uma outra reflexão que gostaria de propor aqui é sobre a contribuição dos números das bases de dados em diferentes narrativas, através das quais os desastres são produzidos enquanto acontecimentos sociais e culturais, e não mais como simples fenômenos naturais. Em um diálogo favorecido pelo ISDR, que financia todas as duas bases, e por artigos de pesquisadores que as comparam 
[24], EM-DAT e Desinventar, temos dois diferentes modos dramáticos, que nos contam sobre dois mundos e seus respectivos desastres.

A primeira narrativa é a planetária. A escala da coleta de dados do EM-DAT é nacional, mas os balanços e, em particular, as traduções destes números (mapas, gráficos de tendências, relatórios, prioridades...) falam, sobretudo, em escala mundial. Fornecendo números que dão a medida de sua amplitude no mundo, EM-DAR serve, em primeiro lugar, para legitimar a necessidade de uma tomada de controle internacional sobre os desastres [25]. Estes números estão alinhados à definição de desastre como "ruptura grave do funcionamento de uma sociedade [...] implicando importantes impactos e perdas [...] que a comunidade ou a sociedade afetada não pode superar somente com seus próprios recursos [26]". A narrativa assim produzida é envolvente. À escala do mundo, veem-se surgir desastres cada vez mais numerosos ou mais destruidores, e mais custosos, embora menos mortíferos [27].

Os mapas e os gráficos traduzem, com cores, esta ideia dramática de urgência no modo pelo qual colorem o mundo em amarelo, laranja e vermelhos de vários tipos, com pontos vermelhos distribuídos sobre continentes ou com gráficos que se erguem em flecha, cujo conjunto constitui uma tentativa de objetivação dos desastres na escala do planeta e, paradoxalmente, criam, no leitor, uma sensação angustiante de excesso. Os desastres estão em todos os lugares, sem cessar de crescer e de produzir efeitos devastadores, é o que nos conta esta narrativa.

Entretanto, este foco narrativo, que fornece os meios para tornar visível a temática dos desastres nas arenas internacionais, constrói, ao mesmo tempo, ângulos cegos e torna certos acontecimentos invisíveis. Deste modo, este foco não permite informar o que, em uma região ou uma cidade específica, pode acentuar os efeitos de um desastre - escolha urbanística, tipos de construção, presença ou não de obras de proteção, vulnerabilidade da população exposta, distribuição de recursos, etc. Centrada sobre as consequências na forma de balanços, e sobre as causas físicas ligadas aos fenômenos naturais, esta narrativa oferece pouco sobre políticas públicas de prevenção.

A narrativa produzida pelo Desinventar é, por sua vez, o negativo 
da primeira e uma tentativa de resposta. É o próprio objeto do “desastre”, que é aí colocado em discurso. Partindo, na maior parte das vezes, não dos perigos, quer dizer dos fenômenos neles mesmos (tempestades, chuvas, terremotos...), mas sobre os efeitos que eles produzem, quer se trate do desmoronamento de uma casa ou do corte de eletricidade em um assentamento informal depois de uma chuva torrencial, os pesquisadores que conduzem este projeto querem tornar visíveis os "pequenos desastres", pois eles sustentam que os efeitos acumulados são, às vezes, mais importantes na escala de um país do que aqueles de um desastre "maior". Crônicos, estes acontecimentos entram, de facto, na categoria de "desastres", a partir de sua inscrição na base de dados. Qualificando-os de "pequenos", "silenciosos", ou ainda, "invisíveis" [28], os pesquisadores que os inserem no Desinventar e aqueles que se utilizam desta base transformam profundamente a compreensão destes desastres.

Contrariamente ao EM-DAT, Desinventar fala de um mundo local, tornado vulnerável pelas políticas de urbanização e de desigualdades flagrantes, regularmente atingido por fenômenos de todos os tipos e povoado por pessoas que enfrentam os perigos cotidianamente. Frequentemente sós ou com a ajuda de serviços locais, bombeiros, voluntários, vizinhos, estas pessoas vivem com a ameaça. Graças ao Desinventar, as suas aflições até então "silenciosas", invisíveis ou inaudíveis, são conduzidas em direção a uma escala que ultrapassa àquela do bairro ou das pequenas cidades. Somadas àquelas de seus vizinhos, suas experiências de infortúnio são incluídas em uma contabilidade geral e podem se transformar em "desastre", mesmo sendo "pequenas". Este único fato lhes permitem ascender ao cenário internacional. Esta narrativa do cotidiano desastroso e do cotidiano reparado, remendado, assumido pelos habitantes, que, sem dúvida, não sabem que vivem um “desastre”, é então amplificada para se tornar audível e objeto de atenção em escala planetária.

Desinventar permite, por sua vez, tornar mais apurada a compreensão destes acontecimentos e de seus impactos, além de oferecer suporte para a ação pública. Esta base de dados pode, por exemplo, sublinhar a recorrência no tempo de fenômenos, que ocorrem em um mesmo território, e permitir de nele concentrar programas para reduzir a sua vulnerabilidade. Se a narrativa que De- 
sinventar produz não é menos inquietante do que aquela originada na base EMDAT, ela é, ao mesmo tempo, mais pragmática e parece oferecer mais direções para as ações.

O antropólogo está mais habituado às narrativas dos habitantes dos bairros ou das pequenas cidades afetados sobre os desastres que viveram. Entretanto, uma atenção aos números que emergem das bases de dados, identificando estes acontecimentos em uma escala mundial ou em uma região do mundo, lhe permite apreender outros tipos de narrativas. Aqui, duas grandes narrativas aparecem em debate e, às vezes, em diálogo uma com outra. Todos as duas tentam falar sobre o mundo e sobre os desastres, mas os focos escolhidos para a observação diferem. A própria noção de "desastre" é posta em discussão, pois se a primeira narrativa tenta evitar o seu transbordamento, ao enquadrá-la em um definição restrita e contável - "ao menos 10 mortes" -; a outra, ao contrário, torna-a acolhedora ao extremo, dando-lhe abertura para integrar os acontecimentos crônicos e rotineiros.

Além das definições, a compilação das grandes quantidades de dados, nas bases das representações dos desastres, com a ajuda de mapas, gráficos e relatórios, por sua vez, narra alguma coisa sobre o nosso mundo: de uma parte, a ameaça crescente, transbordante, mundial; de outra, a ameaça cotidiana e rotineira, que atinge porções do mundo, mas de modo recorrente e silenciosa. Qualquer que seja a narrativa, os números compilados e depois traduzidos vêm apoiar uma operação de colocar em palavras os desastres em escala mundial. Contar os desastres, é também dizê-los.

\section{Notas}

[1] A terminologia geralmente admitida entende assim os acontecimentos produzidos por fenômenos de origem natural: terremotos, tsunamis, furacões, tempestades, episódios de seca ou ondas de frio, erupções vulcânicas. As aspas permitem assinalar que a natureza do fenômeno não está separada da parte da responsabilidade humana nos desastres deste tipo, e, por este motivo, nós as utilizaremos neste texto.

[2] Sandrine Revet, "Penser et affronter les désastres: un panorame des recherches en sciences sociales et des politiques internationales", Critique internationale, n. 52/3, 2011, p. 157-173.

[3] Ver a lista citada pelo CRED: < $\underline{w w w . e m d a t . b e / a d d i t i o n a l-d i s a s t e r-d a t a-r e s o u r c e s ~}>$. 
Acesso em: 6 ago. 2018.

[4] Disponível em: <http://www.emdat.be> . Acesso em: 6 ago. 2018.

[5] Disponível em: <http://desinventar.org > Acesso em: 6 ago. 2018.

[6] Disponível em: <http://www.unisdr.org/we/inform/gar>. Acesso em: 6 ago. 2018.

[7] Daniel Cefaï, "La construction des problèmes publics. Définitions de situations dans des arènes publiques", Réseaux, v. 14, n. 75, 1996, p. 43-66

[8] Entrevista realizada pela autora em Bruxelas, 18 de janeiro de 2011.

[9] EM-DAT não contabiliza unicamente os desastres ditos "naturais", mas igualmente os desastres tecnológicos e biológicos, assim como os conflitos.

[10] Office of U.S. Foreign Disaster Assistance/United States Agency for International Development.

[11] NT: O Crescente Vermelho é a contrapartida da Cruz Vermelha nos países islâmicos.

[12] Debby Guha-Sapir, Philippe Hoyois, Regina Below, Annual Disaster Statistical Review 2013. The Numbers and Trendes, Bruxelles, CRED, 2014.

[13] Debby Guha-Sapir, Femke Vos, Regina Below, Julien Ponserre, Annual Disaster Statistical Review 2010. The Numbers and Trendes, Bruxelles, CRED, 2011.

[14] Lydie Cabane, Sandrine Revet, "La cause des catastrophes. Concurrences scientifiques et actions politiques dans un monde transnational”, Politix, 2015 (no prelo)

[15] Maskrey, Andrew (dir.). Los desastres no son naturales, La Red de Estudios Sociales en Prevencion de Desastres en América Latina, 1993.

[16] Cloé Vallette, Stéphane Cartier, "Dénombrer pour maïtriser les dommages des catastrophes naturelles", VertigO La revue électronique en sciences de l'environnement, v. 12, n. 1, 2012.

[17] Ibid.

[18] Bruno Latour. L'Espoir de Pandore. Pour une version réaliste de l'activité scientifique, Paris, La Découverte, 2001.

[19] Cloé Vallette e Stéphane Cartier citam diversos desastres em que ocorreram disputas sobre o número de vítimas: chuvas torrenciais em Vargas, na Venezuela (Sandrine Revet, Anthropologie d'une catastrophe. Les coulées de boue de 1999 au Venezuela, Paris, Presses Sorbonne Nouvelle, 2007); Xynthia na França (B. De Vanssay, L. Colbeau Justin, D. Marchand, C. Vallette, C. Bakhache, M. Poumadère, "Construire une mémoire sociale utile de la tempête Xynthia du 28 février 2010”, rapport final, Nanterre, Académie de l'eau, 2011, 117 p.); Nargis en Birmanie (Bénédicte Brac de la Perrière, "Le scrutin de Nargis. Le cyclone de 2008 en Birmanie", Terrain, n. 54, "Catstrophes", 2010, p. 6679); inundações do Var na França (Freddy Vinet, Laurent Boissier, Stéphanie Defossez, "La mortalité comme expression de la vulnérabilité humaine face aux catstrophes naturelles"deux inondations récenttes en France (Xynthia, Var, 2010)", VertigO La revue électronique en sciences de l'environnement, v.11, n.2, 2011); acrescenta-se o terremoto no Haiti (Alice Corbet. "Invisibles omniprésents. Les morts du séisme" in Laënnec Hurbon (dir.), Catastrophes et Environnement. Haïti, séisme du 12 janvier 2010, Paris, Éditions de LÉHESS, coll. "Cas de figure", 2014, p. 29-58). Estas disputas são recorrentes e 
muito propagadas, em qualquer que seja o país ou a região do mundo em questão.

[20] Sandrine Revet, Anthropologie d'une catastrophe. les coullés de boue de 1999 au Venezuela, obra citada.

[21] Rogerio Altez, Sandrine Revet, "Contar los muertos para contar la muerte. Discusión en torno al número de fallecidos em la tragedia de 1999 en el estado Vargas" , Revista Geográfica Venezolana, Mérida, Universidad de Los Andes, 2005, numéro spécial, p. 21-43.

[22] Ver Robert D’Ercole, Sébastien Hardy, Jérémy Robert, "Balance de los accidentes y desastres ocurridos en La Paz, Lima y Quito (1970-2007)", Bulletin de l'Institut français d'études andines, v. 38, n.3, 2009, p. 433-465.

[23] Alain Desrosières, La Politique des grands nombres. Histoire de la raison statistique, Paris, La Découverte, 1993.

[24] Cloé Vallette, Stéphane Cartier, "Dénombrer pour maîtriser les dommages des catastrophes naturelles”, artigo citado; Robert D’Ercole, Sébastien Hardy, Jérémy Robert, "Balance de los accidentes y desastres ocurridos en La Paz, Lima y Quito (1970-2007)", artigo citado; Jean-Christophe Gaillard, Ben Wisner, Bernard Nava, "Petites catastrophes et réduction de risques", Humanitaire, n. 38, juillet 2014, p. 60-69; Mabel C. Marulanda, Omar D. Cardona, Alex H. Barbat, "Revealing the Socioeconomic Impact of Small in Columbia Using the Desinventar Database", Disasters, v. 34, n. 2, 2010, p. 552-570.

[25] Sandrine Revet, Vivre dans un monde plus sûr. Catastrophes "naturelles" et sécurité "globale", Cultures \& Conflits, n. 75, hiver 2009, p. 33-51.

[26] Definição do UNISDR. Definição completa: "Ruptura grave do funcionamento de uma comunidade ou de uma sociedade implicando importantes impactos e perdas humanas, materiais, econômicas ou ambientais que a comunidade ou a sociedade afetada não pode superar com seus próprios recursos" (Terminologia para a prevenção dos riscos de desastres, UNISDR, 2009, p. 11, em linha: <www.unisdr.org/files/7817 UNISDRTerminologyFrench.pdf) > . Acesso e: 6 ago. 2018.

[27] Allan Lavell, Andrew Maskrey, “The Future os Disaster Risk Management”, Environmental Hazards, v. 13, n. 4, 2014. Neste artigo originado durante a reunião de grande número de pesquisadores em ciências sociais da corrente "alternativa", cujos numerosos membros e fundadores fazem parte da rede latino-americana La Red, para a preparação da Conferência Mundial sobre a redução de riscos de desastres de 2015, mostrou o paradoxo do "sucesso" das políticas de redução de riscos atuantes há dez anos e fundamentadas em uma melhor preparação, a saber que a mortalidade ocorrida nos fenômenos meteorológicos (chuvas, tempestades, secas...) está em baixa enquanto por outro lado os fenômenos geológicos (do tipo tsunamis ou terremotos) de natureza excepcional são suscetíveis de contradizer esta constatação, assim como os custos dos desastres e seus impactos sobre os modos de vida aumentam sem cessar.

[28] Jean-Christophe Gaillard, Ben Wisner, Bernard Nava, "Petites catastrophes et réduction des risques", artigo citado.

\section{Traduzido do francês por Marta de Araujo Pinheiro (UFRJ)}

